\title{
The Analgesic and Anxiolytic Activity of Resveratrol Mediated by Different Sub-Types of $\alpha$-Adrenoceptors of Anterior Cingulate Cortex Following Neuropathic Pain in Male Rats
}

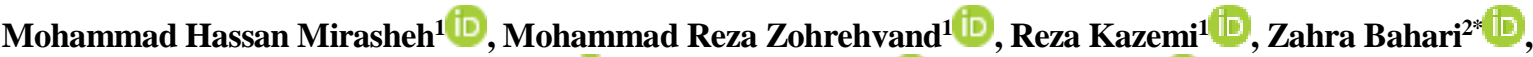 \\ Farideh Bahrami2 $^{(D)}$, Zohreh Jangravi ${ }^{3}\left(\mathbb{D}\right.$, Mehdi Graily ${ }^{2}(\mathbb{D})$
}

1. Students Research Committee, Baqiyatallah University of Medical Sciences, Tehran, Iran

2. Dept. of Physiology and Medical Physics, Faculty of Medicine, Baqiyatallah University of Medical Sciences, Tehran, Iran

3. Dept. of Biochemistry, Faculty of Medicine, Baqiyatallah University of Medical Sciences, Tehran, Iran

\begin{tabular}{|c|}
\hline Article Info \\
\hline d tof $10.30699 /$ jambs.28.129.183 \\
\hline $\begin{array}{l}\text { Received: 2020/01/27; } \\
\text { Accepted: 2020/07/07; } \\
\text { Published Online: } 31 \text { July 2020; }\end{array}$ \\
\hline $\begin{array}{l}\text { Use your device to scan and read the } \\
\text { article online }\end{array}$ \\
\hline 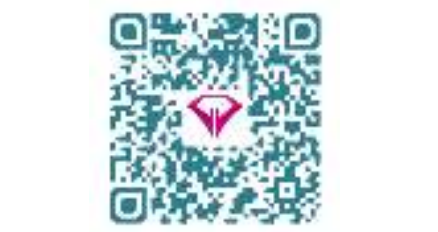 \\
\hline
\end{tabular}

Corresponding Information: Zahra Bahari,

Dept. of Physiology and Medical Physics, Faculty of Medicine, Baqiyatallah University of Medical Sciences, Tehran, Iran

E-Mail: bahari_441@yahoo.com

\begin{abstract}
Background \& Objective: The mechanism of analgesic and anxiolytic activity of resveratrol in neuropathic pain conditions remains obscure. The present study was conducted to examine whether the analgesic and anxiolytic activities of resveratrol are associated with $\alpha_{1}$ - and $\alpha_{2}$-adrenoceptors of the anterior cingulate cortex (ACC), which is a key area of the cortex in the pain process, following neuropathic pain in rats.
\end{abstract}

Materials \& Methods: Neuropathic pain was created by chronic constriction injury (CCI) of the sciatic nerve. Male Wistar rats were assigned to the sham, CCI, CCI+resveratrol (40 $\mu \mathrm{g} / 5 \mu \mathrm{L}$ ), CCI+resveratrol+prazosin ( $\alpha_{1}$-adrenoceptor antagonist, $30 \mu \mathrm{g} / 5 \mu \mathrm{L}$ ), and CCI+resveratrol-Yohimbine ( $\alpha_{2}$-adrenoceptor antagonist, $\left.30 \mu \mathrm{g} / 5 \mu \mathrm{L}\right)$ groups. The rats received intra-ACC injection of the drug on the day of $\mathrm{CCI}$ and for 6 days post-CCI on a daily basis. Cold allodynia (using acetone test) and anxiety (using elevated plus maze, EPM) were examined on days 2,4 , and 6 following CCI.

Results: CCI model significantly increased cold allodynia and anxiety. Resveratrol significantly decreased cold allodynia. Prazosin induced no significant changes in allodynia as compared with the CCI+resveratrol treated group. But the animals in this group had no significant difference from the day before the surgery or compared with the sham group. Prazosin significantly decreased entries into open arms. Additionally, yohimbine significantly increased cold allodynia as compared with the CCI+resveratrol treated group. However, it induced no significant changes in the EPM parameters. Our findings also demonstrated a significant correlation between allodynia and anxiety in CCI rats.

Conclusion: It is suggested that the mechanism of analgesic and anxiolytic activities of resveratrol in the ACC of rats is different, and is mediated through $\alpha_{2}$ and $\alpha_{1}$-adrenoceptors, respectively.

Keywords: Allodynia, Anxiety, Anterior cingulate cortex, $\alpha$-adrenoceptors, Rat, Resveratrol

\section{Introduction}

Peripheral or central nerve damage can induce neuropathic pain $(\mathbf{1}, 2)$. Common abnormal signs of neuropathic pain are hyperalgesia (exaggerated pain perception in response to noxious), allodynia (pain perception in response to non-noxious stimuli) and psychological disorders such as anxiety $(3,4)$. In most of the pharmacological studies along with neuropathic pain management, the effects of different compounds on these behavioral parameters are investigated (5). Nowadays, conventional analgesics and existing treatments only partially alleviate the neuropathic pain, due to its unknown underlying mechanism(s). Moreover, it is suggested that $\alpha$-adrenoceptors critically contribute to the process of pain development (6). These receptors are widely distributed in the central nervous system (7). Several pharmacological studies have originally reported that the administration of $\alpha$ adrenoceptor agonists can attenuate the hypersensitivity that follows neuropathic pain (7-9). For example, Roh et al. (2008) indicated that the intrathecal injection of clonidine (alpha-2 adrenoceptor agonist, $20 \mu \mathrm{g} / \mathrm{rat}$ ) induces anti-hyperalgesic and anti-allodynic effects in the chronic constriction injury (CCI) model of the rats with sciatic nerve (10). Additionally, Liang et al. (2017) revealed that the intrathecal administration of dexmedetomidine attenuated mechanical allodynia and 
thermal hyperalgesia in the CCI model of the sciatic nerve in Sprague-Dawley rats (11). Similarly, Chia et al. (2020) revealed that the administration of zerumbone (the main bioactive compound, a wild ginger plant species) have anti-allodynia and anti-hyperalgesia effects via activation of $\alpha$ - and $\beta$-adrenoceptors in the CCI-induced neuropathic pain mouse model (12). Therefore, it is suggested that $\alpha$-adrenoceptors have an important role in the processing of pain information in the central nervous system. Here, we focus on the $\alpha_{1}$ - and $\alpha_{2}$-adrenoceptors of the anterior cingulate cortex (ACC). Accumulating evidence indicates that the ACC is the most important cortical circuit related to pain information processing (13). Xioe et al. reported that the delivery of repetitive noxious laser to the rat's hind paw induced extracellular activity in the ACC in the rats (14). Additionally, in humans, surgical cingulectomy suppressed responses to noxious stimuli (15). Hence, the activity of neurons in the ACC is associated with pain perception. Resveratrol (3,5,4'-trihydroxystilbene), which is a natural polyphenolic compound, is found in certain fruits and vegetables including red grapes (16). Pharmacological studies have demonstrated that resveratrol possesses diverse properties, including antioxidant, anti-carcinogenic, anti-inflammatory, cell growth-modulatory, and analgesic and anti-nociceptive effects (16-19). Although, the analgesic effects of resveratrol have been well documented, the underlying mechanism of its analgesic and anxiolytic activities is far from clear. Here, we concentrate on the interaction effects of resveratrol with $\alpha_{1}$ - and $\alpha_{2}$-adrenoceptors of the ACC following neuropathic pain among male rats. Indeed, the present study, is aimed to explore whether analgesic or anxiolytic activities of resveratrol are mediated by $\alpha_{1}$ - and $\alpha_{2}$-adrenoceptors of ACC following neuropathic pain.

\section{Materials and Methods}

\section{Animals}

The experiments were carried out on adult male Wistar rats (weight $180 \pm 20 \mathrm{~g}, \mathrm{n}=6$ /group). The animals were purchased from breeding colony of Baqiatallah University of Medical Sciences, Tehran, Iran. The animals were housed in the animal house of Baqiyatallah University of Medical Sciences under a 12-hour light/dark cycle (6 am lights on- 6 pm lights off) in a temperaturecontrolled room (22 $\pm 2^{\circ} \mathrm{Cand}$ humidity: $\left.60 \pm 5 \%\right)$ with food and water available ad libitum. All the experiments were conducted in agreement with the codes of National Institutes of Health Guide for Care and Use of Laboratory Animals. The Ethics Committee of the Baqiatallah University approved this animal study (Ethical code: IR.BMSU.REC.1396.750).

\section{Drugs and Chemicals}

Resveratrol was acquired from Sigma-Aldrich Inc. (St Louis, MO, USA). Prazosin and Yohimbine were purchased from Iran Daru Pharmaceutical Company. The drug was dissolved in physiological saline $(0.9 \%)$.

\section{Experimental Protocol}

In the present study, rats were randomly divided into 5 groups. These groups were as follows: (Group 1: sham-operated group); (Group 2: CCI group); (Group 3: CCI+ Resveratrol group); (Group 4: CCI+ Resveratrol + Prazosin group); and (Group 5: CCI+ Resveratrol + Yohimbine group). Resveratrol (40 $\mu \mathrm{g} / 5$ $\mu \mathrm{L})$ applied intra-ACC daily from days 1 to 6 after surgery ( $n=6$ rats per group). Co-injection Resveratrol and Prazosin (as $\alpha 1$-adrenoceptor antagonist, $30 \mu \mathrm{g} / 5$ $\mu \mathrm{L}$ ) or Yohimbine (as $\alpha 2$-adrenoceptor antagonist, 30 $\mu \mathrm{g} / 5 \mu \mathrm{L}$ ) was also applied daily from days 1 to 6 postsurgery. The behavioral evaluation was carried out 1 day prior to CCI surgery (day -1), and on days 2,4 , and 6 post-surgery (20), at $30 \mathrm{~min}$ after drugs injection.

\section{Drugs Injection}

For intra-ACC injections of normal saline (control), Resveratrol, Prazosin and Yohimbine, a guide cannula (stainless steel 28-gauge) was implanted into the right ACC, contra-lateral to nerve injury, (AP $1.5 \mathrm{~mm}$ from bregma, ML $\pm 0.6 \mathrm{~mm}$ from midline, DV $1.5 \mathrm{~mm}$ beneath the surface of the skull), using stereotaxic frame (21). A 5.0 $\mu \mathrm{L}$ Hamilton syringe with a 33-gaugeneedle was used to inject $5 \mu \mathrm{L}$ of chemical and drugs. The syringe was left in place for 3 min to ensure diffusion of the injected.

\section{Neuropathic Pain Model (CCI Model)}

The left sciatic nerve was tightly ligated to produce the CCI model of neuropathic pain, as previously introduced by Bennett and Xie (22). The rats were anesthetized with chloral hydrate $(350 \mathrm{mg} / \mathrm{kg}$, i.p). The left sciatic nerve (body section of nerve about $1 \mathrm{~cm}$ ) was exposed and 4 loose ligatures (4/0 cat-gut) were tied around the nerve, about $1 \mathrm{~mm}$ apart, until a brief twitch in the hind limb was observed. The muscle and the adjacent fascia and skin were closed with sutures. A sham operation was performed by exposing, but not ligating the sciatic nerve.

\section{Cold Allodynia (Acetone Test)}

To quantify cold sensitivity, the number of paw withdrawal responses (PWR, as a positive response) was evaluated after application of acetone drop to the plantar surface of the left hind paw (injured side) (23). Briefly, the rat was placed under a transparent Plexiglas chamber with a metal mesh floor and an acetone drop was applied to the plantar surface of the hind paw, using a syringe. The acetone drop was applied 5 times (total trials $=5$, every $5 \mathrm{~min}$ ) to the left hind paw. The frequency of PWR was expressed as the percentage and calculated as follows: (Number of positive responses $x$ 100) / (Number of total trials).

\section{Anxiety-like Behaviors (EPM)}

The elevated plus maze is a cross-shaped platform which consists of 2 open and 2 closed arms. All the arms communicate through a central zone. The rats were placed on the central zone of the maze, facing an 
open arm for $5 \mathrm{~min}$. Their movements on the maze were controlled for a $5 \mathrm{~min}$ period by camera. The percentage of time spent in open arms and the percentage of entries into open arms were used as indices of anxious behaviors. A decrease in the time spent in the open arms and the number of entries into open arms indicated anxiety (24).

\section{Statistical Analysis}

All the data were presented as mean \pm standard error of the mean (SEM). Analyses were performed in SPSS 24 (SPSS Inc., Chicago, Ill. USA). Differences in the measured parameters among the 4 groups were analyzed using one-way analysis of variance (ANOVA), followed by Tukey's post-hoc test. Additionally, the data were evaluated using two-way ANOVA to determine the day $\times$ group interaction. Pearson linear regression analysis (variables; frequency of PWR and open arm entries) was also performed between sham and neuropathic group. The differences were considered to be significant atp $<0.05$.

\section{Results}

Effects of Resveratrol and Its Interaction with a1,2-adrenoceptors on Cold Allodynia

The frequency of PWR in CCI rats was significantly higher after the neuropathic surgery as compared to before the surgery (Figure 1). Cold allodynia was significantly increased from 2 days up to 6 days after the neuropathic surgery [Figure $1,\left({ }^{*} P<0.05\right.$ on Day 2$)$ and ( $* * P<0.01$ on Days 4 and 6)]. Additionally, intraACC injection of resveratrol significantly decreased the frequency of PWR as compared with the CCI group [Figure 1, (\#\#\#P<0.001 on Day 4) and (\#\#P<0.01 on Day 6)] (it indicated analgesic activity of Resveratrol). There was no significant difference in PWR between the CCI+ resveratrol and $\mathrm{CCI}+$ resveratrol + prazocin groups. The co-injection of resveratrol and prazosin had no significant effect on the frequency of PWR as compared with the CCI+ resveratrol group. Surprisingly, co-injection of resveratrol and yohimbine significantly increased the frequency of PWR from days 4 to 6 post-surgery as compared with the CCI+ resveratrol group (Figure $1,{ }^{\wedge \wedge} P P<0.001$ and ${ }^{\wedge} P<0.05$; respectively) (that is Yohimbine suppressed analgesic effect of Resveratrol). Two-way ANOVA (days $\times$ groups) analysis confirmed significant effects of groups $(P=0.001)$, and days $(P=0.001)$, as well as interaction between both of the factors $(P=0.002)$.

\section{Effects of Resveratrol and Its Interaction with a1,2-adrenoceptors on Anxiety-Like Behaviors}

In the EPM test, the percentage of entries into open arms decreased on day 6 in CCI rats compared to the sham group; moreover, the percentage of time spent into open arms decreased on all the experimental days after neuropathy in CCI rats as compared with the sham group (Figure 2).

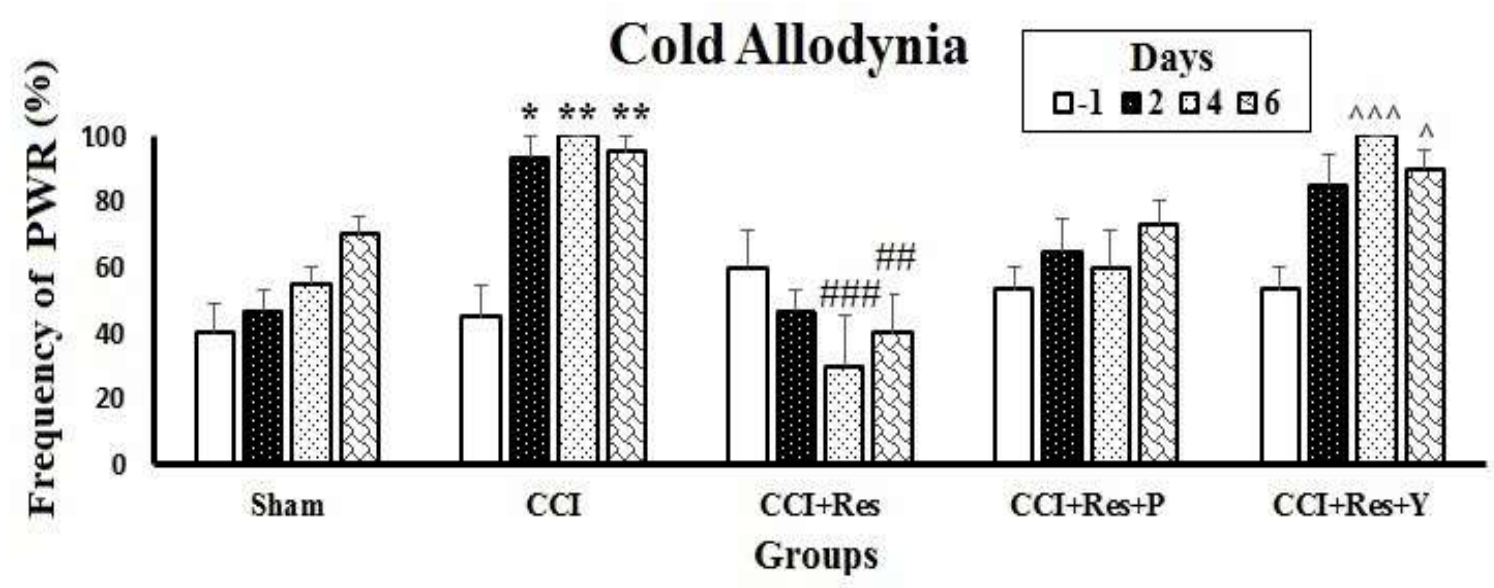

Figure 1. Effects of Resveratrol and its interaction with $\alpha_{1}$ and 2-adrenoceptors on cold allodynia were evaluated. Frequency of paw withdrawal response (PWR) to acetone stimulation was assessed on ipsilateral hind paws of experimental groups, at day -1 (baseline), and days 2,4 , and 6 post-neuropathy. Differences in measured parameters among 4 groups were analyzed using Two- and One-way analysis of variance (ANOVA), followed by the Tukey's posthoc test. CCI; chronic constriction injury, CCI+Res; chronic constriction injury+ Resveratrol, CCI+ Res +P; chronic constriction injury+ Resveratrol + Prazosin, CCI+ Res +Y; chronic constriction injury+ Resveratrol + Yohimbine.

*denote a significant difference with sham animals or day -1 (baseline) in each group; \# denote a significant difference with CCI animals at the same day. ${ }^{\wedge}$ denote a significant difference with CCI+Resveratrol animals at the same day. 


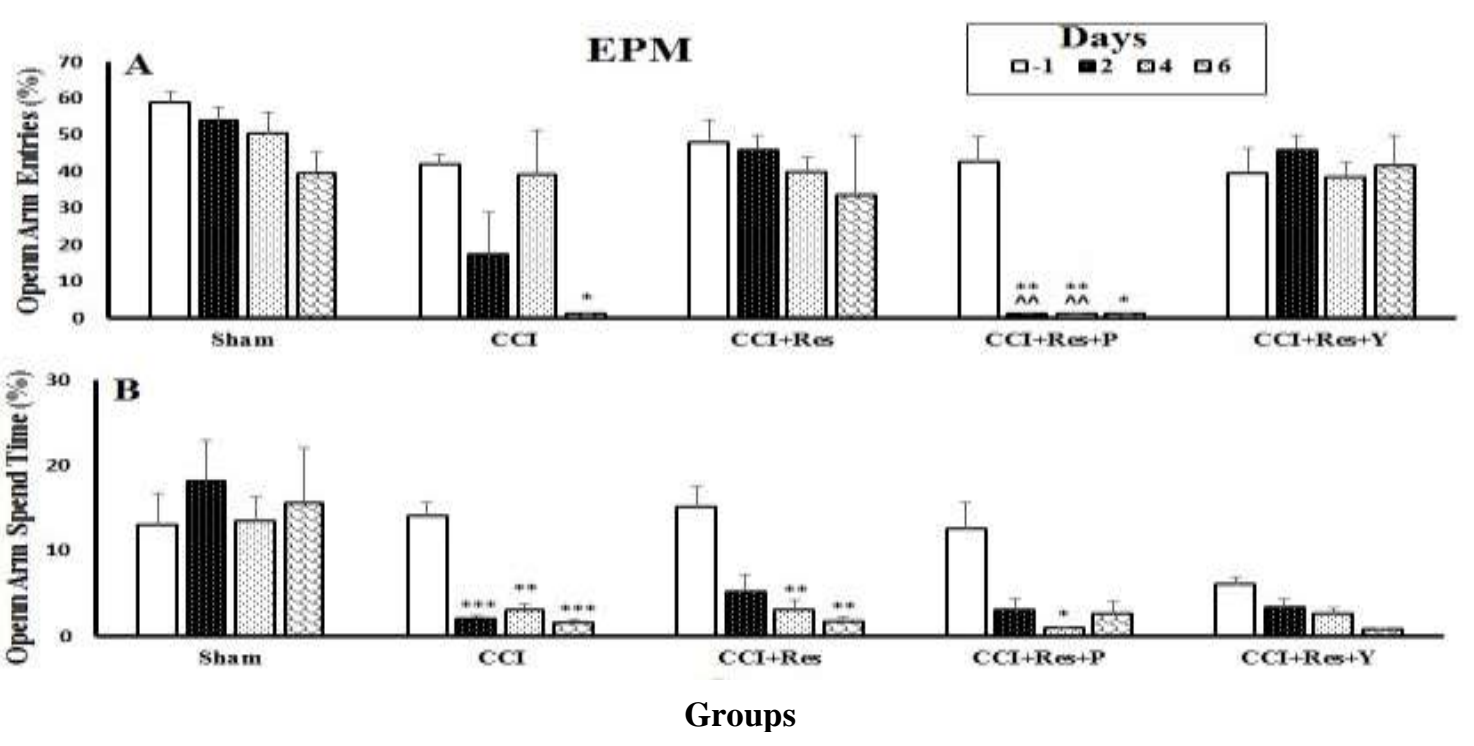

Figure 2. Effects of Resveratrol and its interaction with $\alpha 1$ and 2-adrenoceptors evaluated on anxiety-like behaviors at days -1 (baseline) and 6 post-neuropathy. Percentage of open arms entries (A) and spent time in open arms (B) were evaluated as anxiety indices. Differences in measured parameters were analyzed using two- and one-way analysis of variance (ANOVA), followed by the Tukey's post-hoc test. CCI; chronic constriction injury, CCI+Res; chronic constriction injury+ Resveratrol, CCI+ Res +P; chronic constriction injury+ Resveratrol + Prazosin, CCI+ Res +Y; chronic constriction injury+ Resveratrol + Yohimbine.

* denote a significant difference with sham animals or day -1 (baseline) in each group; ${ }^{\wedge}$ denote a significant difference with CCI+Res animals.
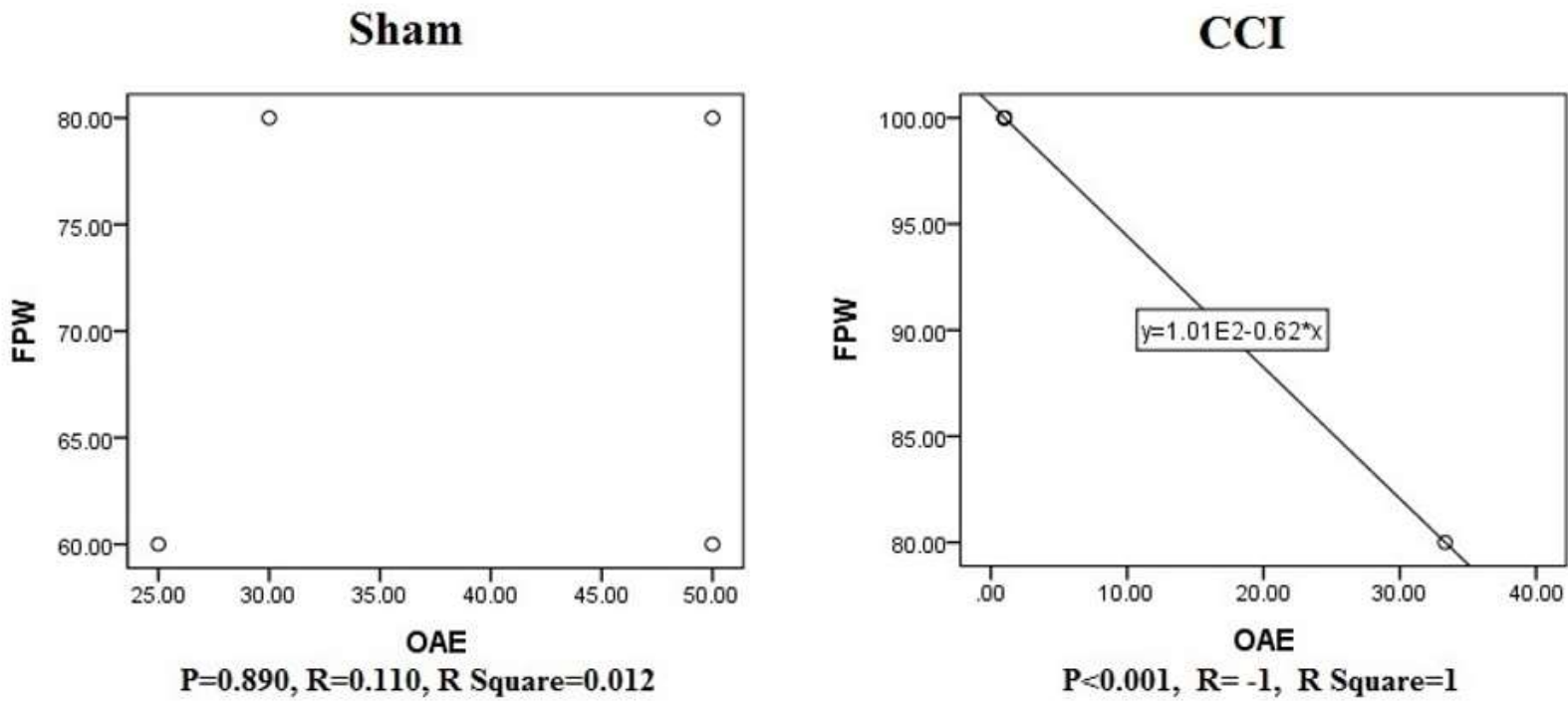

Figure 3. Correlation between allodynia (frequency of paw withdrawal, FPW) and anxiety (open arms entries, OAE) were evaluated in the sham and CCI (neuropathy animals) groups. Data extracted from 6 days after CCI injury. Only significant correlation between allodynia (FPW) and open arm entries was observed in CCI rats. No significant correlation between these parameters was observed in sham-operated rats. The corresponding Pearson correlation (R), $R$ Square, and P-values as determined by regression analysis are indicated below each corresponding panel.

A decline in the percentage of entries into open arms or time spent in open arms displayed increased anxiety. Moreover, intra-ACC injection of resveratrol could not induce a significant alteration in entries into open arms of EPM as compared with the CCI group. However, this increase was not different as compared to before
CCI surgery (day -1) in CCI+ resveratrol group, which is indicated the anxiolytic effects of resveratrol (Figure $2 a)$. In addition, the co-injection of resveratrol and prazosin significantly decreased only the parameter of entries into open arms (increased anxiety) as compared with the CCI+ resveratrol group on days 2 and 4 post- 
surgery (Figure $\left.2,{ }^{\wedge} P P<0.01\right)$. Moreover, there was no significant difference in either of the evaluated parameters of EPM between the CCI+ resveratrol and $\mathrm{CCI}+$ resveratrol +yohimbine groups (Figure 2a). Generally, the co-injection of resveratrol with prazosin or yohimbine did not induce a significant alteration in the percentage of time spent in open arms as compared with the $\mathrm{CCI}+$ resveratrol or CCI groups. The present findings also demonstrated a significant correlation was observed between allodynia (PWR) and anxiety (open arm entries) among the CCI rats (Figure 3; R Square $\left._{\mathrm{CCI}}=1\right)$. No significant correlation was observed between these parameters in sham-operated rats (Figure 3; R Square Sham =0.012). Finally, two-way ANOVA (days $\times$ groups) analysis confirmed the significant effects of groups $(P=0.001)$, and days $(P=0.001)$, as well as interaction between both of the factors $(P=0.002)$.

\section{Discussion}

In the current study, neuropathic pain was induced by tying 4 loose ligatures around the left-side sciatic nerve (CCI model) and a marked cold allodynia was observed in the ipsilateral-hind paw of the animals, which is a hypersensitivity index in the pain pathways, up to 6 days after CCI. No cold allodynia and anxious behavior was observed in the sham-operated rats. Experimental CCI surgery is widely used as a neuropathic pain model that involved in explaining the underlying mechanisms of neuropathic pain (25). Several reports have shown that this different neuropathy model causes allodynia (3,2629). For example, Abbaszadeh et al. demonstrated that the CCI model markedly caused hyperalgesia and allodynia 21 days post-CCI (30). Moreover, in the present study the CCI was found to induce anxiety-like behaviors in the rats, using EPM test. In support of the presents study, Roeska et al. revealed that anxiety-like behaviors markedly increased in the CCI-operated rats (31). In the EPM test, two parameters, including open arms entry and time spent in open arms, were measured as index of anxious behaviors. Indeed, a decline in the open arm entries or open arm time spent indicated increased anxious behaviors in the animal. Anxious behaviors in the EPM are easily assessed and quantified by an observer. The EPM is well-known animal behavioral test and is widely used to evaluate the effects of pharmacological agents and help understand the neuropathic pain mechanism (32). We also demonstrated the significant correlation between allodynia and anxiety (open arm entries) in the CCI rats. No significant correlation was observed between these parameters in the sham-operated rats. Moreover, the present results revealed that intra-ACC injection of resveratrol, as a potent antioxidant, diminished the allodynic activity and anxious behavior (increased percentage of open arms entries parameter in the EPM test, anxiolytic effects) associated with the neuropathic pain. These findings were in accordance with those of previous reports, which have shown the analgesic and anxiolytic effects of resveratrol in the different neuropathy models $(33,34)$. For example, Yin et al. (2013) revealed that the intrathecal administration of resveratrol decreased pain sensitivity in the CCI-induced neuropathic model (35). Similarly, Xu et al. (2018) reported the analgesic effects of the intraperitoneal administration of resveratrol (daily for 14 consecutive days) following CCI surgery (36). In the present study, it is important to note that, the open arms entries parameter was not significantly altered in the CCI group in any of the days of the experiment. Therefore, the present results could suggest that the intra-ACC injection of resveratrol reduced anxious behaviors in the EPM in CCI-operated animals. In our study, we also observed that resveratrol produced no significant alterations in the percentage of spent time of animals in open arms, as another index of anxious behavior, compared with CCI group. It is well accepted that ACC is an important cortical area responsible for process of pain perception (37). Therefore, we selected this area of the brain to investigate the mechanism of analgesic and anxiolytic activity of resveratrol in neuropathic pain. The underlying mechanism(s) of analgesic and anxiolytic activity of resveratrol in neuropathic pain is far from clear. Therefore, one of the main goals of the present study was to investigate the mechanism of analgesic and anxiolytic activity of resveratrol It is well accepted that $\alpha$-adrenoceptors are an important therapeutic target for pain (6). Antidepressants, as the first-line drugs for the treatment of neuropathic pain, inhibit NE and serotonin transporters, and lead to increased norepinephrine levels in the synaptic space (38). In the cortex, pyramidal neurons in many areas of the cortex such as ACC receive a great number of adrenergic inputs from the locus cerulean (39). Koga et al. (2020) reported that the application of NE can induce both pre- and post-synaptic amplification in ACC neurons (39). They also revealed that activation of locus cerulean projection to the ACC increased excitatory transmission in vitro and produced behavioral sensitization for mechanical stimulation (39). Therefore, adrenoceptors significantly contribute to the processing of pain information during neuropathic pain. In the present study, the findings demonstrated that the mechanism of analgesic and anxiolytic activities of resveratrol in the ACC were mediated by different subtypes of receptors. Only concomitant injection of yohimbine with resveratrol significantly suppressed the 
analgesic activity of resveratrol. Indeed, resveratrol seemed to have failed to reduce cold allodynia in the presence of the $\alpha_{2}$-adrenoceptor antagonist in the ACC. Furthermore, concomitant injection of only prazosin with resveratrol significantly attenuated the anxiolytic activity of resveratrol. In fact, resveratrol seems to have failed to increase entries to open arms of EPM in the presence of the $\alpha_{1}$-adrenoceptor antagonist in the ACC.

\section{Conclusion}

The results of the present study revealed that resveratrol exhibited analgesic and anxiolytic effects in the CCI-operated rats. Furthermore, our data have shown that the analgesic and anxiolytic activities of resveratrol were mediated by $\alpha_{2}$ - and $\alpha_{1}$-adrenoceptors in the ACC, respectively. The present study suggests that the mechanism of analgesic and anxiolytic activities of resveratrol in the ACC of rat are different, which are mediated through different subtypes of $\alpha$-adrenoceptors. Our limitation of the present research was lack of determination of the anti-oxidant activity of resveratrol. Further researches are needed to explain the pharmacologic properties of resveratrol in the treatment of the neuropathic pain symptoms.

\section{Acknowledgments}

Present study was supported by Neuroscience Research Center, Baqiyatallah University of Medical Sciences.

\section{Conflict of Interest}

Authors declared no conflict of interest.

\section{References}

1. Alles SR, Smith PA. Etiology and Pharmacology of Neuropathic Pain. Pharmacol Rev. 2018; 70(2):315-47. [DOI:10.1124/pr.117.014399]

2. Bahari Z, Sadr SS, MeftahiGH,et al. Nerve injuryinduced plasticity in the nociceptive pathways. Arch Neurosci. 2015; 2(2):e18214. [DOI:10.5812/archneurosci.18214]

3. Ferreira-Chamorro P, Redondo A, Riego G, Leanez S, Pol O. Sulforaphane inhibited the nociceptive responses, anxiety- and depressivelike behaviors associated with neuropathic pain and improved the anti-allodynic effects of morphine in mice. Front Pharmacol. 2018; 9: 1332. [DOI:10.3389/fphar.2018.01332]

4. Chen H, Hu Y, XieK, et al. Effect of autophagy on allodynia, hyperalgesia and astrocyte activation in a rat model of neuropathic pain. Int $\mathbf{J}$ Mol Med.
2018;

[DOI:10.3892/ijmm.2018.3763]

5. Vakili A, Shirvanian MJ, Safakhah HA, RashidyPour A. Pentoxifylline decreases allodynia and hyperalgesia in a rat model of neuropathic pain. Daru. 2011; 19(4):306-11.

6. Bahari Z, Meftahi GH. Spinal $\alpha 2$-adrenoceptors and neuropathic pain modulation; therapeutic target. Br J Pharmacol. 2019; 176(14):2366-81. [DOI:10.1111/bph.14580]

7. Sudo RT, do Amaral RV, da Silva MonteiroCE,et al. Antinociception induced by a novel $\alpha 2 \mathrm{~A}$ adrenergic receptor agonist in rodents acute and chronic pain models. Eur J Pharmacol. 2017; 815:210-18. [DOI:10.1016/j.ejphar.2017.09.018]

8. Kubre J, Sethi A, Mahobia M, Bindal D, Narang N, Saxena A. Single dose intravenous dexmedetomidine prolongs spinal anesthesia with hyperbaric bupivacaine. Anesth Essays Res. 2016; 10:273-77. [DOI:10.4103/02591162.174465]

9. Sudo RT, Calasans-Maia JA, GaldinoSL,et al. Interaction of morphine with a new alpha2adrenoceptor agonist in mice. J Pain. 2010; 11:718. [DOI:10.1016/j.jpain.2009.08.001]

10. Roh DH, Kim HW, Yoon SY,et al. Intrathecal clonidine suppresses phosphorylation of the $\mathrm{N}$ methyl-D-aspartate receptor NR1 subunit in spinal dorsal horn neurons of rats with neuropathic pain. AnesthAnalg. 2008; 107(2):693-700. [DOI:10.1213/ane.0b013e31817e7319]

11. Liang F, Liu M, Fu X, Zhou X, Chen P, Han F. Dexmedetomidine attenuates neuropathic pain in chronic constriction injury by suppressing NR2B, NF- $\mathrm{BB}$, and iNOS activation. Saudi Pharm J. 2017; 25(4):649-54.

[DOI:10.1016/j.jsps.2017.04.039]

12. Chia JS, Mohammed Izham NA, Farouk AA,et al. Zerumbone modulates $\alpha 2 \mathrm{~A}$-adrenergic, TRPV1, and NMDA NR2B receptors plasticity in CCIinduced neuropathic pain in vivo and LPSinduced SH-SY5Y neuroblastoma in vitro models. Front Pharmacol. 2020; 11: 92. [DOI:10.3389/fphar.2020.00092]

13. Fuchs PN, Peng YB, Boyette-Davis JA, Uhelski ML. The anterior cingulate cortex and pain processing. Front IntegrNeurosci. 2014; 8:35. [DOI:10.3389/fnint.2014.00035]

14. Xiao Z, Martinez E, Kulkarni PM,et al. Cortical pain processing in the rat anterior cingulate cortex and primary somatosensory cortex. Front Cell Neurosci. 2019; 13: 165. [DOI:10.3389/fncel.2019.00165] 
15. Fuchs PN, Peng YB, Boyette-Davis JA, Uhelski ML. The anterior cingulate cortex and pain processing. Front IntegNeurosci. 2014; 8: 35 [DOI:10.3389/fnint.2014.00035]

16. Tao L, Ding Q, Gao C, Sun X. Resveratrol attenuates neuropathic pain through balancing pro-inflammatory and anti-inflammatory cytokines release in mice. IntImmunopharmacol. 2016; $34: 165-72$ [DOI:10.1016/j.intimp.2016.02.033]

17. Cheng W, Zhao Y, Liu H, et al. Resveratrol attenuates bone cancer pain through the inhibition of spinal glial activation and CX3CR1 upregulation. Fund ClinPharmacol. 2014; 28:66170. [DOI:10.1111/fcp.12084]

18. Maia H, Haddad C, Pinheiro N, Casoy J. Advantages of the association of resveratrol with oral contraceptives for management of endometriosis-related pain. Int J Women's Health. 2012; 4:543-49. [DOI:10.2147/IJWH.S36825]

19. Tili E, Michaille JJ. Resveratrol, microRNAs, inflammation, and cancer. J Nucleic Acids. 2011; (2011):102431. [DOI:10.4061/2011/102431]

20. MoiniZanjani T, Ameli H, Labibi F, Sedaghat K, Sabetkasaei M. The attenuation of pain behavior and serum COX-2 concentration by Curcumin in a rat model of neuropathic pain. Korean J Pain. 2014; 27(3): 246-52 [DOI:10.3344/kjp.2014.27.3.246]

21. Um SW, Kim MJ, Leem JW, Bai SJ, Lee BH. Pain-relieving effects of mTOR inhibitor in the anterior cingulate cortex of neuropathic rats. MolNeurobiol. 2019; 56(4):2482-94. [DOI:10.1007/s12035-018-1245-z]

22. Bennett GJ, Xie YK. A peripheral mononeuropathy in rat that produces disorders of pain sensation like those seen in man. Pain. 1988; 33:87-107. [DOI:10.1016/0304-3959(88)902096]

23. Choi Y, Yoon YW, Na HS, Kim SH, Chung JM. Behavioral signs of ongoing pain and cold allodynia in a rat model of neuropathic pain. Pain. 1994; 59:369-76. [DOI:10.1016/03043959(94)90023-X]

24. Akçali D, Belen AD, Babacan A, Bolay H. Nitroglycerin challenge induces lateralized headache in nasociliary nerve-ligated rats: implications for chronic migraine. Turk J Med Sci. 2017; 47:681-88. [DOI:10.3906/sag-160286]

25. Austin PJ, Wu A, Moalem-Taylor G. Chronic constriction of the sciatic nerve and pain hypersensitivity testing in rats. J Vis Exp. 2012; 61: 3393. [DOI:10.3791/3393]
26. Sadeghi M, Manaheji H, Haghparast A, Zaringhalam J, Nazemi S, Bahari Z. Study of the effect of GABAA receptor and glial inhibition on behavioral responses in CCI model of neuropathic pain in rat. Iran South Med Journal .2015; 17(6):1120-34.

27. Murasawa H, Kobayashi H, Saeki K, Kitano Y. Anxiolytic effects of the novel $\alpha 2 \delta$ ligand mirogabalin in a rat model of chronic constriction injury, an experimental model of neuropathic pain. Psychopharmacol.2020;237(1):189-197. [DOI:10.1007/s00213-019-05356-3]

28. Berrocoso E, Mico JA, Vitton O, et al.Evaluation of milnacipran, in comparison with amitriptyline, on cold and mechanical allodynia in a rat model of neuropathic pain. Eur J Pharmacol. 2011; 655(1-3):46-51.

[DOI:10.1016/j.ejphar.2011.01.022]

29. Gambeta E, Batista MA, Maschio GP, Turnes JM, Araya EI, Chichorro JG. Anxiety- but not depressive-like behaviors are related to facial hyperalgesia in a model of trigeminal neuropathic pain in rats. PhysiolBehav. 2018; 191:131-7. [DOI:10.1016/i.physbeh.2018.04.025]

30. Abbaszadeh A, Darabi S, HasanvandA,et al. Minocycline through attenuation of oxidative stress and inflammatory response reduces the neuropathic pain in a rat model of chronic constriction injury. Iran J Basic Med Sci. 2018; 21(2):138-44.

31. Roeska K, Doods H, Arndt K, Treede RD, Ceci A. Anxiety-like behaviour in rats with mononeuropathy is reduced by the analgesic drugs morphine and gabapentin. Pain. 2009; 139:349-57. [DOI:10.1016/j.pain.2008.05.003]

32. Şahin TD, Göçmez SS, Eraldemir FC, Utkan T. Anxiolytic-like and antidepressant-like effects of resveratrol in streptozotocin-induced diabetic rats. NoroPsikiyatrArs. 2019; 56(2): 144-49.

33. Takehana S, Sekiguchi K, Inoue M,et al. Systemic administration of resveratrol suppress the nociceptive neuronal activity of spinal trigeminal nucleus caudalis in rats. Brain Res Bull. 2016; 120:117-22

[DOI:10.1016/j.brainresbull.2015.11.011]

34. Xie JY, Liu S, Wu B,et al. The protective effect of resveratrol in the transmission of neuropathic pain mediated by the $\mathrm{P} 2 \mathrm{X} 7$ receptor in the dorsal root ganglia. Neurochem Int. 2017; 103:24-35. [DOI:10.1016/i.neuint.2016.12.006]

35. Yin Q, Lu FF, Zhao Y,et al. Resveratrol facilitates pain attenuation in a rat model of neuropathic pain through the activation of spinal sirt1. RegAnesth Pain Med. 2013; 38: 93 Y99. [DOI:10.1097/AAP.0b013e3182795b23] 
36. Xu M, Cheng Z, Ding Z, Wang Y, Guo Q, Huang C. Resveratrol enhances IL-4 receptor-mediated anti-inflammatory effects in spinal cord and attenuates neuropathic pain following sciatic nerve injury. Mol Pain. 2018; 14: 1-11. [DOI:10.1177/1744806918767549]

37. Zhao R, Zhou H, Huang L, et al.Neuropathic pain causes pyramidal neuronal hyperactivity in the anterior cingulate cortex. Front Cell Neurosci. 2018; 12:107. [DOI:10.3389/fncel.2018.00107]
38. Obata H. Analgesic mechanisms of antidepressants for neuropathic pain. Int $\mathrm{J}$ Mol Sci. 2017; 18(11): E2483. [DOI:10.3390/ijms18112483]

39. Koga K, Yamada A, Song Q,et al. Ascending noradrenergic excitation from the locus coeruleus to the anterior cingulate cortex. Mol Brain. 2020; 13:49. [DOI:10.1186/s13041-020-00586-5]

\section{How to Cite This Article:}

Mirasheh M H, Zohrehvand M R, Kazemi R, Bahari Z, Bahrami F, Jangravi Z et al . The Analgesic and Anxiolytic Activity of Resveratrol Mediated by Different Sub-Types of $\alpha$-Adrenoceptors of Anterior Cingulate Cortex Following Neuropathic Pain in Male Rats. J Adv Med Biomed Res. 2020; 28 (129) :183-190

\section{Download citation:}

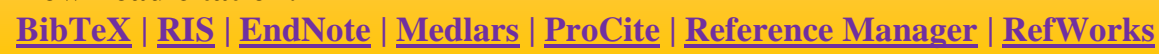

\section{Send citation to:}

S. Mendeley Zotero OPefWorka RefWorks 